\title{
Incidence, risk factors, timing, and outcome of influenza versus COVID-19-associated putative invasive aspergillosis
}

\author{
Camille Sarrazyn MD¹, Sofie Dhaese MD PhD, Birgit Demey², Stefaan Vandecasteele MD PhD ${ }^{1}$, Marijke Reynders MD ${ }^{3}$ \\ and Jens T. Van Praet MD PhD ${ }^{1,4}$ (i) \\ ${ }^{1}$ Department of Nephrology and Infectious Diseases, AZ Sint-Jan Brugge-Oostende AV, Brugge, Belgium, ${ }^{2}$ Faculty of Medicine and Health Sciences, Ghent \\ University, Ghent, Belgium, ${ }^{3}$ Department of Laboratory Medicine, Medical Microbiology, AZ Sint-Jan Brugge-Oostende AV, Brugge, Belgium and ${ }^{4}$ Department of \\ Internal Medicine and Pediatrics, Ghent University, Ghent, Belgium
}

To the Editor-Invasive pulmonary aspergillosis (IPA) is a wellestablished superinfection of influenza, especially in critically ill patients admitted to the intensive care unit (ICU). ${ }^{1}$ The incidence of this complication depends on the definition used and environmental factors, and classical host risk factors for IPA are generally absent in these patients. More recently, several reports have emerged of coronavirus disease 2019 (COVID-19)-associated IPA. ${ }^{2-4}$ However, whether the risk and morbidity of IPA is comparable between both viral illnesses remains unclear. We compared the incidence, risk factors, timing, and outcome of influenza and COVID-19-associated IPA for the 2019-2020 season in our institution, a 1,182-bed acute- and tertiary-care hospital in Belgium consisting of 3 separate campuses.

To study influenza-associated IPA, we included all consecutively admitted outpatients between October 1, 2019, and March 30, 2020, who met the World Health Organization (WHO) severe acute respiratory infection (SARI) case definition with a positive combined nasopharyngeal or throat swab for influenza RNA, tested by real-time (RT)-PCR on Taqman array cards $(n=141$, median age, 66 years; interquartile range [IQR], 47-78; 47\% female). Overall, 92 patients had influenza A H1N1; 33 had influenza A H3N2; 5 had influenza B; and 11 patients had influenza that could not be typed due to the low viral concentration.

For the analysis of COVID-19-associated IPA, we collected the data for all consecutively admitted outpatients between March 11, 2020, and April 17, 2020, with a positive nasopharyngeal swab (RT-PCR testing) for and/or IgG antibodies (MPIA on Architect-I System from Abbott, Sligo, Ireland) against severe acute respiratory coronavirus virus 2 (SARS-CoV-2) upon admission $(n=131$; median age, 67 years; IQR, 56-79; 40\% female). For the definition of IPA, we used 'putative IPA' because the optimal criteria for COVID-19-associated IPA have not been established. ${ }^{5}$ This definition requires the identification of Aspergillus spp in culture of bronchoalveolar lavage fluid or meeting at least 2 of the following conditions: the identification of Aspergillus spp in a culture of bronchial aspirate, positive galactomannan detection in bronchial aspirate or bronchoalveolar

\footnotetext{
Author for correspondence: Dr Jens T. Van Praet, E-mail: Jens.VanPraet@azsintjan.be Cite this article: Sarrazyn C, et al. (2021). Incidence, risk factors, timing, and outcome of influenza versus COVID-19-associated putative invasive aspergillosis. Infection Control \& Hospital Epidemiology, 42: 1149-1150, https://doi.org/10.1017/ice.2020.460
}

lavage fluid (Platelia antigen EIA from Bio-Rad, Berkeley, CA, with a cut-off index of $>1.00$ ) or positive RT-PCR for Aspergillus spp in bronchial aspirate or bronchoalveolar lavage fluid. The latter 2 tests are routinely performed in our laboratory on all lower respiratory tract samples. For COVID-19 patients, the microbiological tests were performed on sputum or bronchial aspirate, which was routinely collected 3 times weekly from ventilated patients, given the risk of aerosolization during bronchoscopy. ${ }^{6}$ Patients were considered at risk for IPA the first 28 days after diagnosis of viral illness, or until death. Negative binomial regression was used to calculate the incidence and $95 \%$ confidence interval of IPA in patients with influenza or COVID-19.

Putative IPA was diagnosed in 5 patients of the influenza cohort (3.5\%) and 4 of the COVID-19 cohort (3\%), conferring to incidences of 218.7 per 100 patient years (95\% CI, 40.1$1,627.9$ ) and 118.8 per 100 patient years (95\% CI, 21.6-834.2), respectively. All influenza types of the patients with influenzaassociated IPA were A/H1N1. The clinical and microbiological characteristics of both patient groups are shown in Table 1. The time between admission and diagnosis of IPA was not different: a median of 6 days for influenza versus 4 days for COVID-19. All patients with COVID-19-associated IPA died, compared to 3 out of 5 influenza-associated IPA patients; all of these patients were ventilated in the ICU.

In this single-center analysis, we found a similar incidence of IPA as superinfection of influenza and COVID-19. However, IPA was only observed in COVID-19 patients who needed mechanical ventilation, whereas IPA was also seen in influenza patients hospitalized outside the ICU. As described previously, most influenza and all COVID-19 patients lacked the classical host risk factors for IPA in our series. The timing of the IPA diagnosis was also comparable in both groups. We observed a very poor prognosis of both influenza and COVID-19-associated IPA in our ICU patients. Notably, galactomannan detection is not validated on non-bronchoalveolar lavage fluid respiratory samples, which might have led to overdiagnosis of putative IPA in COVID-19 patients. Nevertheless, our findings support the implementation of active surveillance for IPA early after admission in mechanically ventilated COVID-19 patients.

\section{Acknowledgments.}

Financial support. No financial support was provided relevant to this article.

Conflicts of interest. All authors report no conflicts of interest relevant to this article. 
Table 1. The Clinical and Microbiological Characteristics of Patients With Influenza or COVID-19-Associated IPA During the 2019-2020 Season

\begin{tabular}{|c|c|c|}
\hline Characteristic & $\begin{array}{l}\text { Influenza } \\
\text { Patients }\end{array}$ & COVID-19 Patients \\
\hline Age, median y (range) & $73(66-79)$ & $75(67-89)$ \\
\hline Sex, male & $4 / 5$ & $3 / 4$ \\
\hline Positive galactomannan ${ }^{a}$ & $5 / 5$ & $4 / 4$ \\
\hline Positive $\mathrm{PCR}^{\mathrm{a}}$ & $5 / 5$ & $2 / 4$ (2 tests ND) \\
\hline $\begin{array}{l}\text { Positive culture for Aspergillus } \\
\mathrm{spp}^{\mathrm{a}}\end{array}$ & $3 / 5$ & $4 / 4$ \\
\hline $\begin{array}{l}\text { Immunocompromised according } \\
\text { due to EORTC/MSG criteria }\end{array}$ & $2 / 5$ & $0 / 4$ \\
\hline Hematological malignancy & $1 / 5$ & $0 / 4$ \\
\hline Neutropenia & $2 / 5$ & $0 / 4$ \\
\hline COPD & $4 / 5$ & $0 / 4$ \\
\hline $\begin{array}{l}\text { Steroid use within } 28 \text { days } \\
\text { before admission }\end{array}$ & $2 / 5$ & $0 / 4$ \\
\hline $\mathrm{BMI}>30$ & $2 / 5$ & $2 / 4$ \\
\hline CKD stage III or higher & $1 / 5$ & $1 / 4$ \\
\hline ICU admission & $3 / 5$ & $4 / 4$ \\
\hline Mechanical ventilation & $3 / 5$ & $4 / 4$ \\
\hline Antifungal treatment & $\begin{array}{l}\text { Voriconazole and caspofungin }(n=1) \text {, voriconazole } \\
(n=3) \text {, death before treatment initiation }(n=1)\end{array}$ & $\begin{array}{l}\text { Voriconazole }(n=1) \text {, voriconazole followed by liposomal } \\
\text { amphotericin } B(n=2) \text {, death before treatment initiation }(n=1)\end{array}$ \\
\hline
\end{tabular}

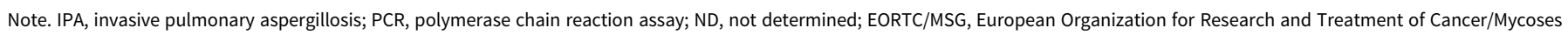
Study Group; COPD, chronic obstructive pulmonary disease; BMI, body mass index, CKD, chonic kidney disease, ICU, intensive care unit.

${ }^{a}$ These tests were performed on lower respiratory tract samples.

\section{References}

1. Rijnders BJA, Schauwvlieghe A, Wauters J. Influenza-associated pulmonary aspergillosis: a local or global lethal combination? Clin Infect Dis 2020. doi: org/10.1093/cid/ciaa010.

2. van Arkel ALE, Rijpstra TA, Belderbos HNA, van Wijngaarden P, Verweij PE, Bentvelsen RG. COVID-19-associated pulmonary aspergillosis. Am J Respir Crit Care Med 2020;202:132-135.

3. Rutsaert L, Steinfort N, Van Hunsel T, et al. COVID-19-associated invasive pulmonary aspergillosis. Ann Intensive Care 2020;10:71.
4. Bartoletti M, Pascale R, Cricca M, et al. Epidemiology of invasive pulmonary aspergillosis among COVID-19 intubated patients: a prospective study. Clin Infect Dis 2020. doi: org/10.1093/cid/ciaal065.

5. Alanio A, Delliere S, Fodil S, Bretagne S, Megarbane B. Prevalence of putative invasive pulmonary aspergillosis in critically ill patients with COVID-19. Lancet Respir Med 2020;8:e48-e49.

6. Van Biesen S, Kwa D, Bosman RJ, Juffermans NP. Detection of invasive pulmonary aspergillosis in COVID-19 with non-directed bronchoalveolar lavage. Am J Respir Crit Care Med 2020. doi: 10.1164/rccm.2020052018LE.

\title{
Aerosolized SARS-CoV-2 transmission risk: Surgical or N95 masks?
}

\author{
Petrick Periyasamy MMed ${ }^{1}$, B. H. Ng MMed ${ }^{1}$, Umi K. Ali MPath², Zetti Z. Rashid MPath² and Najma Kori MMed ${ }^{1}$ (1) \\ ${ }^{1}$ Department of Medicine, Universiti Kebangsaan Malaysia Medical Centre, Kuala Lumpur, Malaysia and ${ }^{2}$ Department of Medical Microbiology and Immunology, \\ Universiti Kebangsaan Malaysia Medical Centre, Kuala Lumpur, Malaysia
}

To the Editor-Based on available evidence, coronavirus disease 2019 (COVID-19) is thought to spread through close contact

Author for correspondence: Dr Najma Kori, Department of Medicine, Level 8, UKM Medical Centre, Jalan Haji Yaakob Latif, Cheras, 56000, Kuala Lumpur. E-mail: najmakori@ukm.edu.my

Cite this article: Periyasamy $\mathrm{P}$, et al. (2021). Aerosolized SARS-CoV-2 transmission risk: Surgical or N95 masks?. Infection Control \& Hospital Epidemiology, 42: 1150-1152, https://doi.org/10.1017/ice.2020.465 and droplet transmission. However, some have debated that it could be airborne. Airborne transmission occurs when particles of $<0.5 \mu \mathrm{m}$ within droplets spread through exhaled air via a process called aerosolization. These particles can remain in the air for long periods and can disseminate over distances $>1 \mathrm{~m}$. In the context of COVID-19, airborne particles can occur during certain aerosolgenerating procedures (AGPs). The World Health Organization (WHO) underlines the use of $\mathrm{N} 95$ respirators or equivalent as part

(c) The Society for Healthcare Epidemiology of America 2020. This is an Open Access article, distributed under the terms of the Creative Commons Attribution licence (http://creativecommons.org/licenses/by/4.0/), which permits unrestricted re-use, distribution, and reproduction in any medium, provided the original work is properly cited. 